\title{
Comparison of Driving Performance on the Unimanual and Bimanual Steering during Simulated Driving between Post-Stroke Patients and Healthy Adults
}

\author{
Han Seong Choe, Kwan Sub Lee, Hwa Kyung Shin \\ Department of Physical therapy, The Catholic University of Daegu, Daegue, Korea \\ hkshin1@cu.ac.kr
}

\begin{abstract}
This study was aimed to analyze the driving performance on the bimanual and unimanual steering during the simulated driving after stroke. Forty five patients with stroke and healthy adults were recruited for this study. 15 subjects were healthy adults and 20 subjects were stroke patients. A virtual reality (VR) driving simulation system (UC-win/Road Ver .5) was used as the experimental apparatus for driving task. Participants steered with paretic, non-paretic and both arms in the straight road of simulator. Main outcome measures were offset from the Lane On Center(LOC). In the main experiment, the participants drove on the $5 \mathrm{~km}$ straight road 3 times, the average of which was used for analysis. Repeated two-way ANOVA was used to compare the groups and Boneferroni was employed for a post-hoc test. The RMSE(Root Mean Square Error) showed a significant difference by the use of the hand and group $(\mathrm{p}<0.05)$. It is thought that bimanual driving is better to activate more brain neurons in post stroke hemiparetic patients and that using both bimanual and unimanual driving is beneficial for the patients to use the hemiparetic side and improve overall driving performance. More driving rehabilitation and visual field training are needed for the left hemiparetic patients who have right hemisphere damage.
\end{abstract}

Keywords: Driving performance, Steering, Stroke, Bimanual, Unimanual.

\section{Introduction}

Hemiparesis is a common impairment occurring after stroke and causes various motor, visual, cognitive, perceptive and sensory deficits. This is also the cause of motor and functional impairment of the upper and lower extremities and affects driving ability[1]. The movement of vehicle for driving depends on the behaviors of a driver. Of these behaviors, steering is the basic and important behavior of the driver. Steering generally requires coordination of both hands. Impairment from hemiparesis disrupts the efficient use of both hands. This disruption leads to avoiding the use of the affected side through a compensatory strategy, which in turn results in inefficient and slow movement[2]. Post-stroke patients require a strategy for coordination and voluntary use of both hands. In order to measure the degree of impairment in a setting to move the steering wheel after stroke, quantification is made for force, position, strength, movement time, reaction time and movement speed of the hands[3]. Most of previous studies focused on the assessment of driving ability using the force and position of the hands. This study attempted to assess driving ability according to the use of the hand by measuring the accuracy of steering and speed of a vehicle in a virtual steering environment. Driver's ability was objectively assessed using a designated scenario, through which the basic driving ability of a driver can be measured. Driving ability was assessed using individual data obtained through a virtual screen.

\section{Methods}

\section{Subjects}

Forty five subjects participated in the experiment. Of the subjects, 15 subjects were healthy adults and 20 subjects were stroke patients. Of the stroke patients, 10 subjects were right hemiplegic and 10 subjects were left hemiplegic patients. Inclusion criteria were: (1) at least 1 year from the onset of stroke (2) absence of ankle joint flexion contracture (3) possession of a valid state driver's license. Exclusion criteria were: (1) severe spasticity of the ankle (modified Ashworth's scale: $>2$ ) or tremor (2) visual problem to see the sine waves are displayed on a PC monitor at $80 \mathrm{~cm}$ distance or severe cognitive impairments (scoring $<25$ on the Mini-Mental State Examination), (3) visual problem or severe cognitive impairment (scoring $<25$ on the Mini-Mental State Examination). Informed consent form was obtained from the all subjects prior to study.

\section{Apparatus \& Procedure}

In the UC-win/Road (Forum8, Japan) almost similar to the real driving environment, minimize errors, the program can be measured to digital map data, satellite data and 3D model data. This study used a simulator, including main computer, 27-inch monitor, automatic gear, and three-wheel pedal. Before starting experiment, the subjects were requested to perform practice driving for 5 minutes so that they would be adapted to the simulator driving environment. In the main experiment, the participants drove on the $5 \mathrm{~km}$ straight road 3 times, the average of which was used for analysis. When a verbal cue of "go" was given, the subjects started driving the car, maintaining the speed at $60 \mathrm{~km}$. In this 
study, the Lane on Center (LOC) was measured from the UC-win/Road.

\section{Statistical Analysis}

In the UC-win/Road, the Lane on Center (LOC), an indicator for how a vehicle is deviated from the road, was measured. Also, the ability to maintain steering with the steering wheel was assessed by calculating the Root Mean Square Error(RMSE), which is used to obtain the absolute error in the continuous tasks, in order to measure the accuracy of continuous skill performance, such as tracking tasks[4].

$$
\text { RMSE }=\sqrt{\frac{\sum_{\mathrm{i}=1}^{\mathrm{n}}\left(\mathrm{yi}^{\wedge}-\mathrm{yi}\right)^{2}}{\mathrm{n}}}
$$

SPSS 17.0 for Windows (SPSS Inc, Illinois) were used for all data analyses. Repeated two-way ANOVA was employed to compare the paretic, non-paretic and both hands. Bonferroni was used as a post-hoc test to compare inter-group difference $(p<0.05)$

\section{Results}

The RMSE of LOC showed a significant difference by the use of the hand and group $(\mathrm{p}<0.05)$, but no interaction was found between the use of the hand and group ( $>0.05)$ (Table 2). It was found from the post-hoc test using Bonferroni that there was a significant difference between non-paretic and paretic hand, and between paretic hand and both hands, depending on the use of the hand $(p<0.05)$. It was also shown from the groups that there was a significant difference between healthy adults and left-hemiparetic patients, and between right hemiparetic patients and left hemiparetic patients $(\mathrm{p}<0.05)$.

Table 1. RMSE values

\begin{tabular}{ccc}
\hline Group & Hand & Mean \pm SD \\
\hline \hline \multirow{3}{*}{ Healthy } & Non-paretic & $10.93 \pm 5.05$ \\
\cline { 2 - 3 } & Paretic & $11.93 \pm 3.62$ \\
\cline { 2 - 3 } & Both & $11.32 \pm 4.76$ \\
\hline \multirow{3}{*}{ Right Hemi } & Non-paretic & $13.59 \pm 6.61$ \\
\cline { 2 - 3 } & Paretic & $15.63 \pm 5.75$ \\
\cline { 2 - 3 } & Both & $14.77 \pm 6.79$ \\
\hline \multirow{3}{*}{ Left Hemi } & Non-paretic & $32.04 \pm 13.03$ \\
\cline { 2 - 3 } & Paretic & $36.72 \pm 12.63$ \\
\cline { 2 - 3 } & Both & $30.52 \pm 12.30$ \\
\hline
\end{tabular}

Table 2. RMSE results

\begin{tabular}{cccccc}
\hline Variables & SS & df & $\begin{array}{c}\text { Mean } \\
\text { square }\end{array}$ & F & p \\
\hline \hline hand & 147.78 & 2 & 73.89 & 4.27 & $0.02^{*}$ \\
\hline group & 9101.30 & 2 & 4550.65 & 28.17 & $0.00^{* *}$ \\
\hline
\end{tabular}

\begin{tabular}{cccccc}
\hline $\begin{array}{c}\text { hand }^{*} \\
\text { group }\end{array}$ & 112.37 & 4 & 28.09 & 1.62 & 0.18 \\
\hline & & & ${ }^{*} \mathrm{p}<.05,{ }^{* *} \mathrm{p}<.01$
\end{tabular}

\section{Conclusion}

The reason that the RMSE of LOC is different by the use of the hand is due to the fact that coordination improves in the setting where both hands are used[3, 5, 6] and to handness, where the preferred hand is used more to perform tasks[7], and to the fact that the upper extremity function of the affected side decreases after brain injury. Results from an electrophysiological study showed that the neurons of supplementary motor area (SMA) were connected for the movement of the same and the opposite sides. It was also found from the comparison between bimanual movement and unimanual movement that the neurons of SMA became more active in bimanual movement. In addition, it was revealed that the similar result was obtained in the primary motor regions $[8,9]$. It is thought that bimanual driving would be better to activate more brain neurons of hemiparetic patients after stroke. It was suggested that the kinematic function of the affected arm improves when performing the same symmetrical movement $[10$, 11]. It will be helpful for post-stroke hemiparetic patients to perform both bimanual driving and unimanual driving for the improvement of the use of the affected side and overall driving performance. The reason the RMSE varied by group was due to the difference in driving performance by brain damage lesion. It was shown that, in general, the driving performance of patients with right hemisphere damage is considerably lower. In an assessment for the visual information after stroke, it was found that more burdens were given on visual attention, visual processing, speed, and visual scanning skills. Damage on the temporoparietal lobe in the right hemisphere indicates the deficit of spatial perception ability. The deficit of spatio-temporal perception ability seriously affects driving ability. Also, patients with right hemisphere damage exhibits neglect. Patients with visual field damage and left neglect tend to forget or neglect the person or object on the left. The present experiment also revealed that patients with right hemisphere damage could not maintain the vehicle in the center of the road, with more rocking, while driving. Based on the results of this experiment, it is thought that visual field training is essential for the driving rehabilitation of hemiplegic patients after stroke and that, in particular, more driving rehabilitation and visual field training are needed for the left hemiparetic patients who have right hemisphere damage from stroke. It is thought that further study needs to assess driving performance through various driving scenarios in the actual environment with real vehicles. 


\section{Acknowledgements}

This research was supported by Basic Science Research Program through the National Research Foundation of Korea(NRF) funded by the Ministry of Education, Science and Technology(No. 20139079)

\section{References}

[1] Carey YJ., Kimberley TJ., Lewis SM.., et al.:Analysis of fMRI and finger tracking training in subjects with chronic stroke, Brain, 235(4): 773-788, 2002

[2] Charles J. and Gordon A. M.:Development of hand-arm bimanual intensive training (HABIT) for improving bimanual coordination in children with hemiplegic cerebral palsy, Developmental Medicine \& Child Neurology, 48(11):931-936, 2006

[3] Jones. RD., Donaldson IM.., Sharman NB.:A Technique for Removal of the Visuoperceptual Its Application to Parkinson's Disease Component from Tracking Performance and Its Application to Parkinson's Disease, IEEE Transactions on Biomedical Engineering, 43:1001-1010, Oct 1996

[4] Kim SJ.:Motor Learning and Control, 4th ed.:34-39, Daehan media, 2013

[5] Johnson MJ., Van der Loos HFM., Burgar CG., et al.:Experimental Results using Force-Feedback Cueing in Robot-Assisted Stroke Therapy, IEEE Transactions on Neural Systems and Rehabilitation Engineering, 13(3):335-348, 2005

[6] Nair P., Jadhav C., Krovi V.:Development and Testing of a LowCost Diagnostic Tool for Upper Limb Dysfunction, IEEE RSJ, 2600-2605, 2003.

[7] Corey DM., Hurely MM., Foundas AL.:Right and left handness defined: A multivaraite approach using hand preference and hand performance measures, Neuropsychiatry, Neuropsychol Behav Neurol, 14(3):144-152, 2001

[8] Brinkman C. and Porter R.:Supplementary motor area in the monkey:activity of neurons during performance of a learned motor task.J, Neurophysiol, 42:681-709, 1979

[9] Tanji J., Okano K., Sato KC.:Neuronal activity in cortical motor areas related to ipsilateral, contralateral, and bilateral digit movements of the monkey, J. Neurophysiol, 60:325-343, 1988

[10] Sugden D. and Utley A.:Interlimb coupling in children with hemiplegic cerebral palsy, Developmental Medicine and Child Neurology, 37(4):293-309, 1995

[11] Utley A. and Sugden D.:Interlimb coupling in children with hemiplegic cerebral palsy during reaching and grasping at speed, Developmental Medicine and Child Neurology, 40(6):396-404, 1998 